\title{
E. coli 086 O-Antigen Biosynthetic Gene Cluster and Stepwise Enzymatic Synthesis of Human Blood Group B Antigen Tetrasaccharide
}

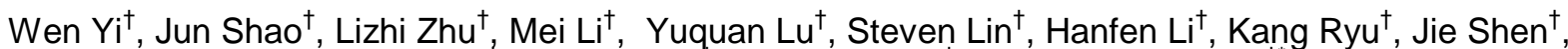 \\ Hongjie Guo ${ }^{\dagger}$, Qingjia $\mathrm{Yao}^{\dagger}$, C. Allen Bush ${ }^{\ddagger}$ and Peng G. Wang \\ $\dagger$ Department of Biochemistry, the Ohio State University, Ohio 43210, and $\$$ Department of Chemistry, University of \\ Maryland, Baltimore County, Maryland 21250
}

\section{Supporting Information}

Contents:

Bacterial strains and plasmids

Shotgun sequence of $E$. coli O86:K62:H2 O-antigen gene cluster

Cloning, expression and purification of glycosyltransferases

Glycosyltransferase activity assay

Enzymatic synthesis of blood group B tetrasaccharide

NMR data for step-wise enzymatic synthesized products 


\section{Bacteria strains and plasmids}

E. coli O86:K62:H2 strain was kindly provided by W. F. Vann from Department of Health and Human Service, Food and Drug Administration. E. coli competent cell DH5 $\alpha$ [lacZ $4 M 15$ hsdR recA] was from Gibco-BRL Life Technology. E. coli competent cell BL21 (DE3) $\left[\mathrm{F}^{-}\right.$ompT hsdS $S_{B}\left(r_{B}{ }^{-} m_{B}{ }^{-}\right)$ gal dcm (DE3)] were from Novagen Inc. (Madison, WI).

\section{Shotgun sequence of $E$. coli O86:K62:H2 O-antigen gene cluster}

The chromosomal DNA of E. coli O86 was prepared by ELU-QUIK DNA purification kit from Schleicher \& Schuell Bioscience, Inc. (Keene, NH). Long-range PCR was carried out by using MasterAmp ${ }^{\mathrm{TM}}$ extra-long PCR kit from Epicentre (Madison, WI). A pair of primers: F (5'CTCTCTGAATACTCCGTCATC) and R (5'ACCTGCTTTCACCATTAACAGGAT) were designed based on the highly conserved sequences of E. coli galF and gnd genes, respectively. A total of 10 PCR products were pooled to limit the PCR errors. After gel purification, the PCR products were sheared by nebulizers (Invitrogen, Carlsbad, CA) and cloned into pCR4Blunt-TOPO vector to generate a bank (260 colonies) according to the instruction manual of TOPO Shotgun Cloning Kit (Invitrogen, Carlsbad, CA). The plasmids for sequencing were prepared with QIAprep 96 Turbo Miniprep kit by using the BioRobot 9600 of Qiagen, Inc. (Valencia, CA). Sequencing was carried out by an Applied Biosystems 3700 DNA Analyzer (Foster City, CA) at the Ohio state University DNA sequencing facility. The Phred/Phrap package from the Genome Center of the University of Washington was used for sequencing assembling. The open reading frames were recognized and selected by using the ORF Finder from the National center for Biotechnology Information (NCBI). The BLAST database was used to search for sequence homologies and the amino acid sequence alignments were performed using CLUSTAL W. Potential transmembrane segments were identified by TMpred. 


\section{Cloning, expression and purification of putative glycosyltransferases}

The $w b n I, w b n J$ and $w b n K$ genes were amplified by PCR from the E. coli O86:K62:H2 chromosome. The primers with rescriction sites underlined for amplification each gene were as follows:

( $\boldsymbol{w} \boldsymbol{b} \boldsymbol{n} \boldsymbol{I}) \mathrm{B} 1$ 5' CCGCCATATGGTTATTAATATATTTT (NdeI)

/B2 5' GCACACTCGAGTTACTTCTTGATATTACCA (Xhol);

( $\boldsymbol{w} \boldsymbol{b} \boldsymbol{n} \boldsymbol{J})$ D1 5' GATATACATATGTCATTGAGAATATTAGAT (NdeI)/D2 5'

CCGCCTCGAGTTATTTTATTAGTGCTTTAC (XhoI);

( $\boldsymbol{w} \boldsymbol{b} \boldsymbol{n} \boldsymbol{K})$ P1 5'CGCGGATCCATGGAAGTTAAAATTATTGGGGGGCT (BamHI)/ P2 5'

CGTTCTCGAGTCATAATTTTACCCACGATTCG $(X h o l)$

The DNA fragments obtained were digested with corresponding restriction enzymes and inserted into pGEX-4T-1 or pET15b vectors linearized by the same restriction enzymes to form pGEX-4T-1wbnK, pET15b-wbnI and pET15b-wbnJ plasmids. The recombinant plasmids were confirmed by restriction mapping and sequencing. The correct constructs were subsequently transformed into $E$. coli BL21 (DE3) for protein expression. E. coli BL21 (DE3) harboring the recombinant plasmid was grown in $1 \mathrm{~L}$ Luria-Bertani (LB) medium at $37^{\circ} \mathrm{C}$. Isopropyl-1-thio- $\beta$-D-galactospyranoside (IPTG) was added to a final concentration of $0.8 \mathrm{mM}$ for pGEX-wbnK and $0.4 \mathrm{mM}$ for pET15b-wbnI and pET-15b-wbnJ. Expression was allowed to proceed for $8 \mathrm{~h}$ at $30^{\circ} \mathrm{C}$. Cells were harvested and stored at $-80{ }^{\circ} \mathrm{C}$ until needed. In protein purification, we used two different affinity columns. In the case of WbnK, the cell pellet was suspended in GST bind buffer (4.3 mM Na $2 \mathrm{HPO}_{4}, 1.47 \mathrm{mM} \mathrm{KH}_{2} \mathrm{PO}_{4}, 137$ $\mathrm{mM} \mathrm{NaCl}, 2.7 \mathrm{mM} \mathrm{KCl}, \mathrm{pH}$ 7.3) and disrupted by sonication on ice. After centrifugation, the lysate was loaded onto a GST-Bind column (1ml, Invitrogen). After washing with the same buffer for four times, the protein was eluted with the elution buffer $(50 \mathrm{mM}$ Tris- $\mathrm{HCl}, 10 \mathrm{mM}$ reduced Glutathione, $\mathrm{pH}$ 8.0). In the case of $\mathrm{WbnI}$ and $\mathrm{WbnJ}$, The cell pellet was resuspended in chilled lysis buffer (50 $\mathrm{mM}$ Tris-HCl, pH 7.0, 0.1 M NaCl, 0.5\% (w/v) Triton X-100, 10\% glycerin (w/v), and $10 \mathrm{mM} 2-$ mercaptoethanol) and disrupted by brief sonication (Branson Sonifier 450, VWR Scientific) on ice. The lysate was cleared by centrifugation $\left(12,000 \mathrm{~g}, 20 \mathrm{~min}, 4{ }^{\circ} \mathrm{C}\right.$ ) and loaded at a low rate of 2 $\mathrm{ml} / \mathrm{min}$ onto a $\mathrm{Ni}^{2+}$-NTA (nickel-nitrilotriacetic acid) agarose affinity column $(15 \mathrm{~mL}$ bed volume, Qiagen, CA) equilibrated with $50 \mathrm{mM}$ Tris- $\mathrm{HCl}, \mathrm{pH} 8.7,400 \mathrm{mM} \mathrm{NaCl}$, and $5 \mathrm{mM}$ imidazole. The column was washed with 5 column volumes of $20 \mathrm{mM}$ imidazole in the same buffer, and the protein was eluted with $200 \mathrm{mM}$ imidazole. Protein eluted from the column was analyzed by SDS-PAGE. 


\section{Glycosyltransferase activity assay}

Standard assay for glycosyltransferase activity is essentially the same with the variation of sugar donor and acceptor in terms of the individual enzymes. Protein concentration is determined by Bradford method. For all the enzymatic assays, 1 unit of enzyme activity was defined as the amount of enzyme required to transform $1 \mu \mathrm{mol}$ of sugar donor to acceptor per minute at $37^{\circ} \mathrm{C}$. Enzyme assays were performed at $37^{\circ} \mathrm{C}$ for $2 \mathrm{hr}$ in a final volume of $100 \mu \mathrm{L}$ containing $20 \mathrm{mM}$ Tris-HCl, $\mathrm{pH}$ 7.0, $10 \mathrm{mM} \mathrm{MnCl}$, $1 \mathrm{mM}$ ATP, $0.3 \mathrm{mM}$ radioactively labeled sugar donor, $10 \mathrm{mM}$ acceptor and variable amount of enzymes. Acceptor was omitted as the blank control. The $\beta 1,3-$ galactosyltransferase $(W b n J)$ was assayed using $0.3 \mathrm{mM}$ radioactively labeled UDP-D-[6$\left.\mathrm{H}^{3}\right]$ Galactose $(10000 \mathrm{cpm})$ as sugar donor, and $10 \mathrm{mM}$ monosaccharide (GalNAco-OMe) as sugar acceptor. The $\alpha 1$,2-fucosyltransferase $(W b n K$ ) was assayed using $0.3 \mathrm{mM}$ radioactively labeled GDP-L-[U- $\left.{ }^{14} \mathrm{C}\right]$ fucose $(7000 \mathrm{cpm})$ as sugar donor, and $10 \mathrm{mM}$ synthesized disaccharide (Gal $\beta 1,3$ GalNAc $\alpha-O M e)$ as sugar acceptor. The $\alpha 1,3$-galactosyltransferase ( $W b n I)$ was assayed using 0.3 $\mathrm{mM}$ radioactively labeled UDP-D-[6-H3]Galactose $(10000 \mathrm{cpm})$ as sugar donor, and 10mM trisaccharide (Fuc $\alpha 1,2-\mathrm{Gal} \beta 1,3-\mathrm{GalNAc} \alpha-\mathrm{OMe}$ ) as sugar acceptor. The reaction was terminated by adding 100ul ice cold 0.1M EDTA. Dowex 1× 8-200 chloride anion exchange resin was then added in a water suspension $(0.8 \mathrm{~mL}, \mathrm{v} / \mathrm{v}=1 / 1)$. After centrifugation, supernatant $(0.45 \mathrm{~mL})$ was collected in a $20 \mathrm{~mL}$ plastic vial and ScintiVerse BD $(10 \mathrm{~mL})$ was added. The vial was vortexed thoroughly before the radioactivity of the mixture was counted in a liquid scintillation counter (Beckmann LS3801 counter).

\section{Step-wise enzymatic synthesis of blood group B tetrasaccharide}

(i). Synthesis of disaccharide Gal $\beta 1,3-$ GalNAc $\alpha$-OMe using WbnJ protein. One pot reaction was conducted for three days at $30^{\circ} \mathrm{C}$ in a final volume of $4.0 \mathrm{ml}$ containing $20 \mathrm{mM}$ Tris- $\mathrm{HCl}(\mathrm{pH} 7.5)$, $10 \mathrm{mM} \mathrm{MnCl}_{2}, 1 \mathrm{mM}$ dithiothreitol (DTT), $10 \mathrm{mM}$ UDP-Gal, and $15 \mathrm{mM}$ acceptor GalNAco-OMe, and $15 \mathrm{mU} \mathrm{WbnJ}$ protein. The progress of the reaction was monitored by thin-layer chromatography $\left[\mathrm{i}-\mathrm{PrOH} / \mathrm{H}_{2} \mathrm{O} / \mathrm{NH}_{4} \mathrm{OH}=8: 2: 2(\mathrm{v} / \mathrm{v} / \mathrm{v})\right]$ conducted on Baker Si250F silica gel TLC plates. Products were visualized by staining solution (anisaldehyde/MeOH/H${ }_{2} \mathrm{SO}_{4}=1: 15: 2(\mathrm{v} / \mathrm{v} / \mathrm{v})$ ). After complete conversion of donor substrate, protein was removed by brief boiling and centrifugation $(12,000 \mathrm{~g}$, 
5min). The supernatant mixture was purified by gel filtration chromatography Bio-Gel P2 (Bio-Rad, CA). The desired fractions were pooled, lyophilized, and stored at $-20^{\circ} \mathrm{C}$.

(ii) Synthesis of trisaccharide Fuc $\alpha 1,2-G a 1 \beta 1,3-G a l N A c \alpha-O M e$ using WbnK/GST fusion protein. The reaction process was the same as mentioned before in disaccharide synthesis with the exception that the donor is $10 \mathrm{mM}$ GDP-Fuc and acceptor is $13 \mathrm{mM}$ disaccharide (Gal $\beta 1,3-\mathrm{GalNAc} \alpha-\mathrm{OMe})$ obtained previously.

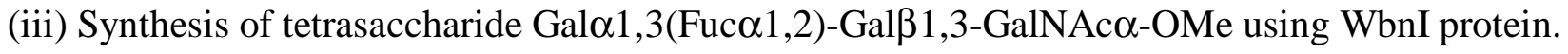
The total reaction volumn was $1 \mathrm{ml}$ containing $20 \mathrm{mM}$ Tris- $\mathrm{HCl}(\mathrm{pH} 7.5), 10 \mathrm{mM} \mathrm{MnCl} 2,1 \mathrm{mM}$ dithiothreitol (DTT), 18 mM UDP-Gal, and 20 mM acceptor trisaccharide (Fuc $\alpha 1,2-G a 1 \beta 1,3-$ GalNAco-OMe), and 20mU WbnI protein.

Mass spectra (ESI) were run in the negative model at the mass spectrometry facility at the Ohio state University. Product structure was identified by ${ }^{1} \mathrm{H}$ and ${ }^{13} \mathrm{C}$ NMR spectroscopy using $500-\mathrm{MHz}$ Varian VXR500 NMR spectrometer. Product Structure was identified through one-dimensional (selective COSY, relay COSY, and NOE) and two-dimensional (COSY, HMQC, NOESY, and HMBC) ${ }^{1} \mathrm{H}-/{ }^{13} \mathrm{C}$ - NMR. The oligosaccharide product was repeatedly dissolved in $\mathrm{D}_{2} \mathrm{O}$ and lyophilized before NMR spectra were recorded at $303 \mathrm{~K}$ in a $5 \mathrm{~mm}$ tube. 
NMR data for step-wise enzymatic synthesized products

\section{Disaccharide $\beta$-D-Gal $p-(1 \rightarrow 3)-\alpha$-Gal $p$ NAc-OMe}

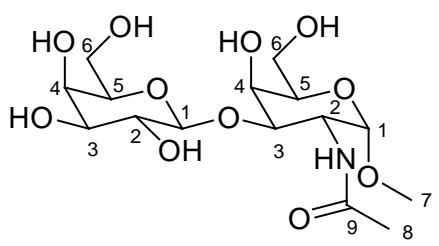

Exact Mass(M+Na)+: 420.1476

Found: $\quad 420.1474$

\begin{tabular}{|c|c|c|c|c|}
\hline \multirow[b]{2}{*}{ \# } & \multicolumn{2}{|l|}{ Gal } & \multicolumn{2}{|l|}{ GalNAc } \\
\hline & ${ }^{1} \mathrm{H}$ NMR & ${ }^{13} \mathrm{C} \mathrm{NMR}$ & ${ }^{1} \mathrm{H}$ NMR & ${ }^{13} \mathrm{C}$ NMR \\
\hline 1 & $4.41, \mathrm{~d}, \mathrm{~J}=7.8 \mathrm{~Hz}, 1 \mathrm{H}$ & 105.12 & $4.74, \mathrm{~d}, \mathrm{~J}=3.7 \mathrm{~Hz}, 1 \mathrm{H}$ & 98.74 \\
\hline 2 & $\begin{array}{l}3.47, \mathrm{dd}, \mathrm{J}=9.9,7.8 \mathrm{~Hz}, \\
1 \mathrm{H}\end{array}$ & 70.86 & $\begin{array}{l}4.29, \mathrm{dd}, \mathrm{J}=11.1,3.7 \\
\mathrm{~Hz}, 1 \mathrm{H}\end{array}$ & 49.01 \\
\hline 3 & $\begin{array}{l}3.57, \mathrm{dd}, \mathrm{J}=9.9,3.4 \mathrm{~Hz} \text {, } \\
1 \mathrm{H}\end{array}$ & 72.97 & $\begin{array}{l}3.96, \mathrm{dd}, \mathrm{J}=11.1,3.1 \\
\mathrm{~Hz}, 1 \mathrm{H}\end{array}$ & 77.72 \\
\hline 4 & $3.86, \mathrm{~d}, \mathrm{~J}=3.3 \mathrm{~Hz}, 1 \mathrm{H}$ & 69.01 & $4.18, \mathrm{~d}, \mathrm{~J}=2.9 \mathrm{~Hz}, 1 \mathrm{H}$ & 69.17 \\
\hline 5 & $\begin{array}{l}3.61, \mathrm{dd}, \mathrm{J}=7.7,4.6 \mathrm{~Hz}, \\
1 \mathrm{H}\end{array}$ & 75.39 & $\begin{array}{l}3.91, \mathrm{dd}, \mathrm{J}=7.3,5.0 \mathrm{~Hz}, \\
1 \mathrm{H}\end{array}$ & 71.04 \\
\hline 6 & $3.71, \mathrm{~m}, 2 \mathrm{H}$ & 61.39 & $3.72, \mathrm{~m}, 2 \mathrm{H}$ & 61.65 \\
\hline 7 & & & $3.35, \mathrm{~s}, 3 \mathrm{H},(\mathrm{OMe})$ & 55.52 \\
\hline 8 & & & $1.98, \mathrm{~s}, 3 \mathrm{H}\left(\mathrm{COCH}_{3}\right)$ & 22.44 \\
\hline 9 & & & $\mathrm{C}=\mathrm{O}$ & 175.02 \\
\hline
\end{tabular}




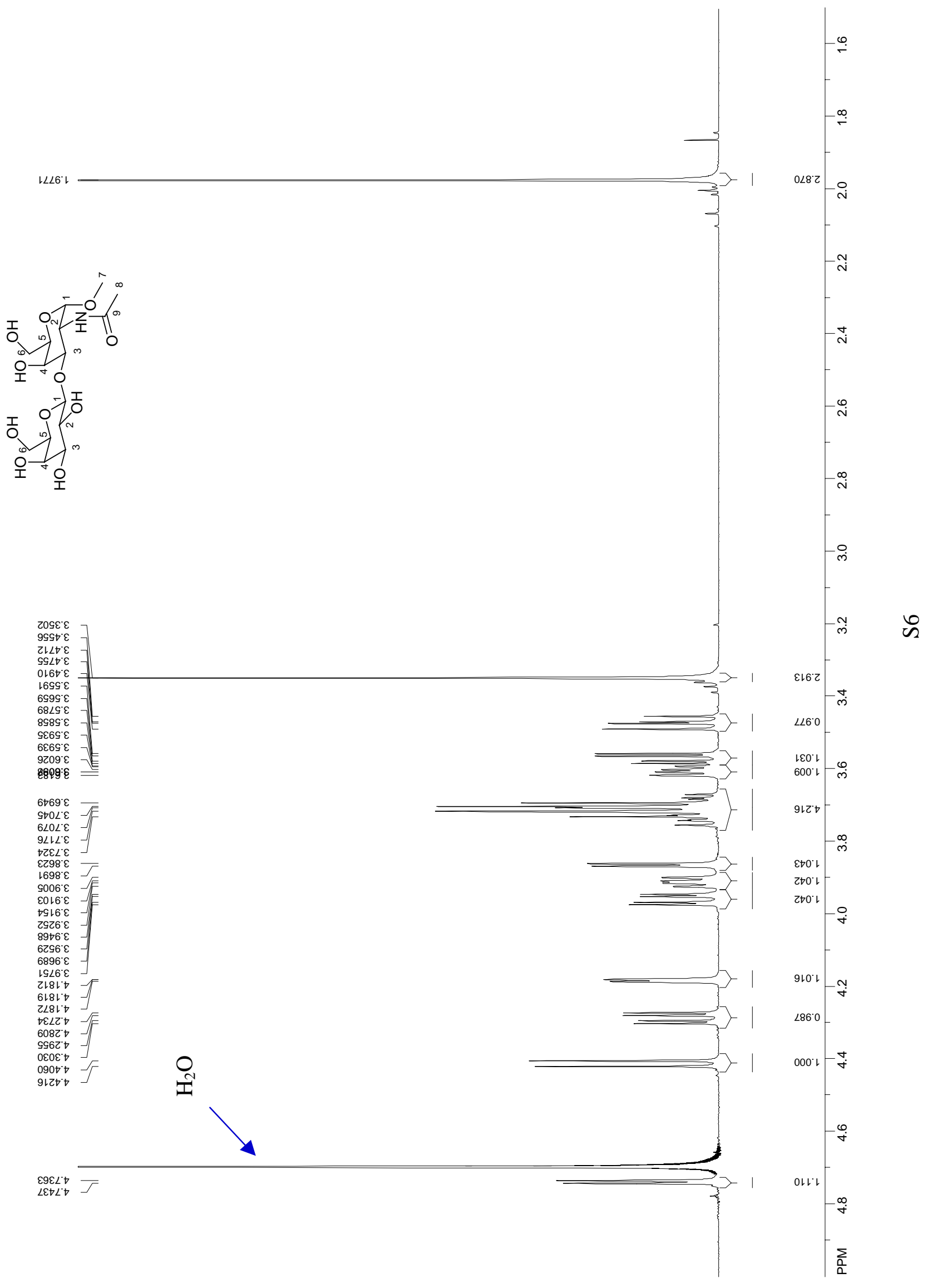


ง6\&t'Z2 -

งะเ 06 -

stटs's -

เ68E. 19
$1+99.19$

\$800.69 -

เ89.69

COOt0. L J

$888 \varepsilon^{\prime} \mathrm{SL}-$

ILL'LL -

乙StL'86 -

SLZI' SOL -

ט.

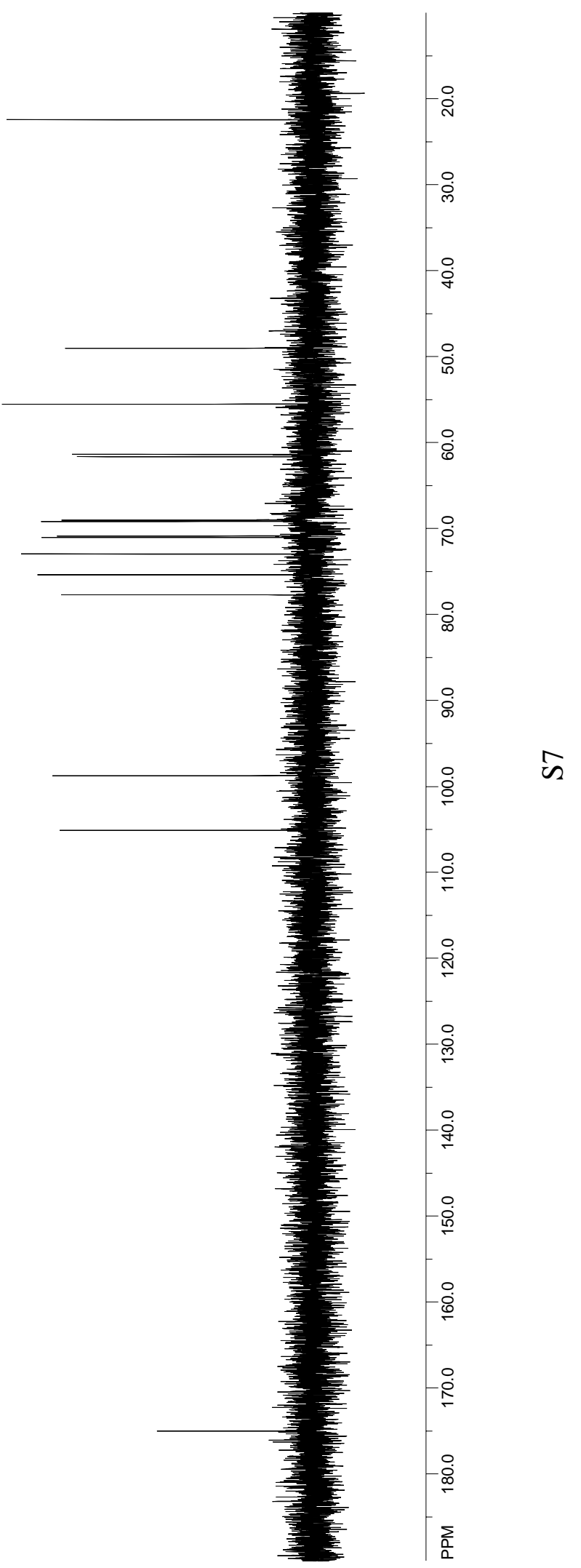

$\stackrel{ }{=}$ 


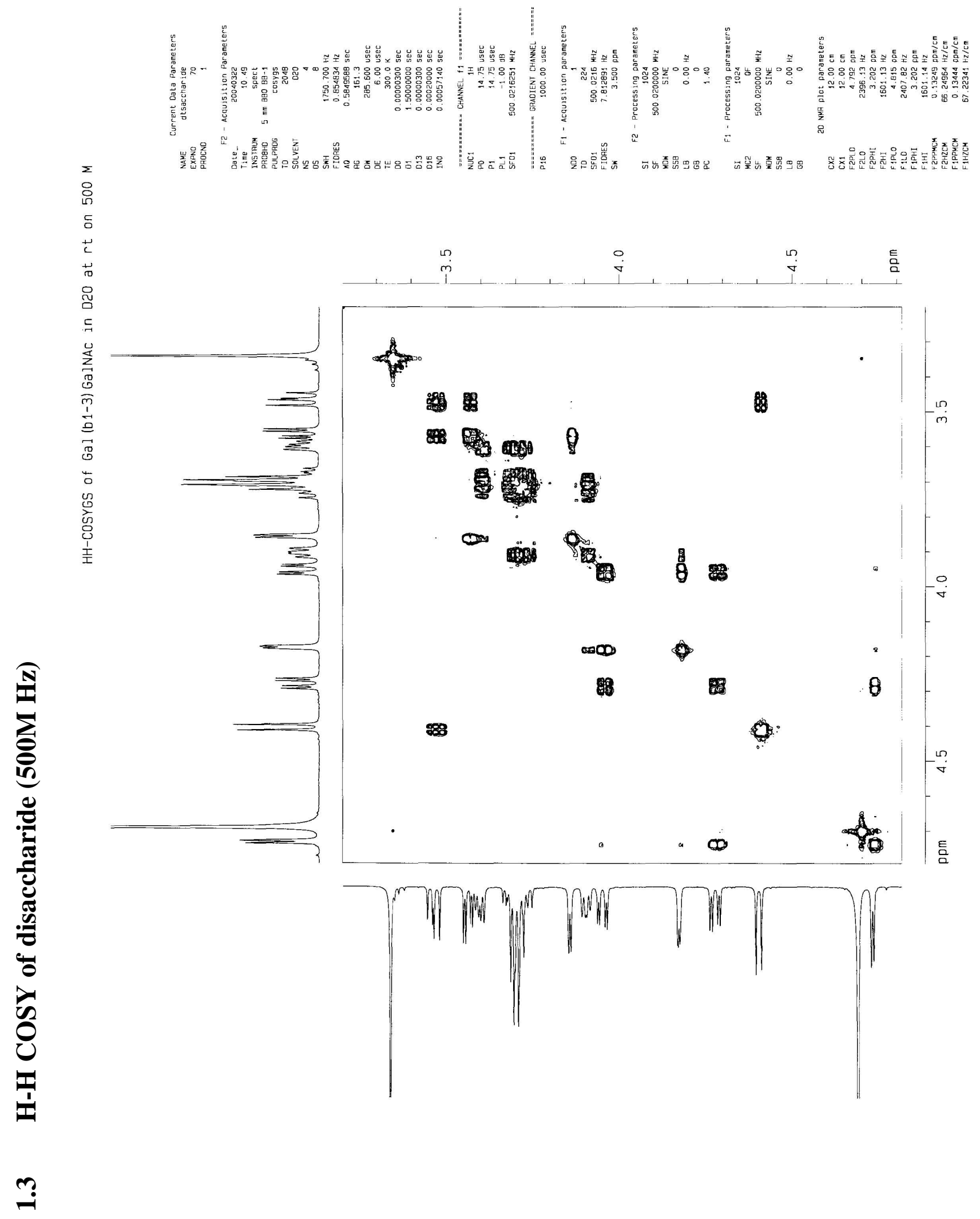




\section{Trisaccharide $\alpha$-L-Fucp $(1 \rightarrow 2)$ - $\beta$-D-Gal $p-(1 \rightarrow 3)-\alpha$-Gal $p$ NAc-OMe}

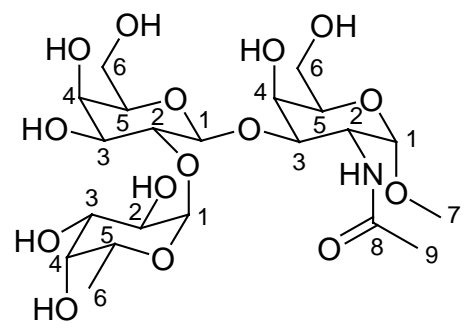

\begin{tabular}{|l|l|l|l|l|l|l|}
\hline & \multicolumn{2}{|c|}{ GalNAc } & \multicolumn{2}{c|}{ Gal } & \multicolumn{2}{c|}{ Fuc } \\
\hline$\#$ & \multicolumn{1}{|c}{${ }^{1} \mathrm{H}(\delta)$} & ${ }^{13} \mathrm{C}(\delta)$ & \multicolumn{1}{c|}{${ }^{1} \mathrm{H}(\delta)$} & ${ }^{13} \mathrm{C}(\delta)$ & \multicolumn{1}{l}{$\mathrm{H}(\delta)$} & ${ }^{13} \mathrm{C}(\delta)$ \\
\hline 1 & $4.96, \mathrm{~d}, J=3.2 \mathrm{~Hz}, 1 \mathrm{H}$ & 97.87 & $4.81, \mathrm{~d}, J=7.7 \mathrm{~Hz}, 1 \mathrm{H}$ & 101.96 & $5.41, \mathrm{~d}, J=3.6 \mathrm{~Hz}, 1 \mathrm{H}$ & 99.28 \\
\hline 2 & $4.37, \mathrm{~m}, 1 \mathrm{H}$ & 49.39 & $3.82, \mathrm{~m}, 1 \mathrm{H}$ & 74.99 & $3.95, \mathrm{~m}, 1 \mathrm{H}$ & 68.07 \\
\hline 3 & $4.28, \mathrm{dd}, J=11.1,2.7 \mathrm{~Hz}$, & 73.76 & $4.01, \mathrm{dd}, J=9.7,3.1 \mathrm{~Hz}$ & 73.54 & $3.86, \mathrm{~m}, 1 \mathrm{H}$ & 71.85 \\
\hline 4 & $4.36, \mathrm{~m}, 1 \mathrm{H}$ & 69.05 & $3.84, \mathrm{~m}, 1 \mathrm{H}$ & 69.55 & $3.81, \mathrm{~m}, 1 \mathrm{H}$ & 76.28 \\
\hline 5 & $4.13, \mathrm{t}, J=5.8 \mathrm{~Hz}, 1 \mathrm{H}$ & 70.39 & $4.09, \mathrm{~d}, J=3.0 \mathrm{~Hz}, 1 \mathrm{H}$ & 69.05 & $4.39, \mathrm{~m}, 1 \mathrm{H}$ & 66.78 \\
\hline 6 & $3.95, \mathrm{~m}, 2 \mathrm{H}$ & 61.22 & $3.96, \mathrm{~m}, 2 \mathrm{H}$ & 60.90 & $1.38, \mathrm{~d}, J=6.5 \mathrm{~Hz}, 3 \mathrm{H}$ & 15.34 \\
\hline 7 & $3.55, \mathrm{~s}, 3 \mathrm{H}\left(\mathrm{OCH}_{3}\right)$ & 55.09 & & & & \\
\hline 8 & $\mathrm{C}=\mathrm{O}$ & 173.66 & & & & \\
\hline 9 & $2.22, \mathrm{~s}, 3 \mathrm{H}\left(\mathrm{COCH}_{3}\right)$ & 21.93 & & & & \\
\hline
\end{tabular}



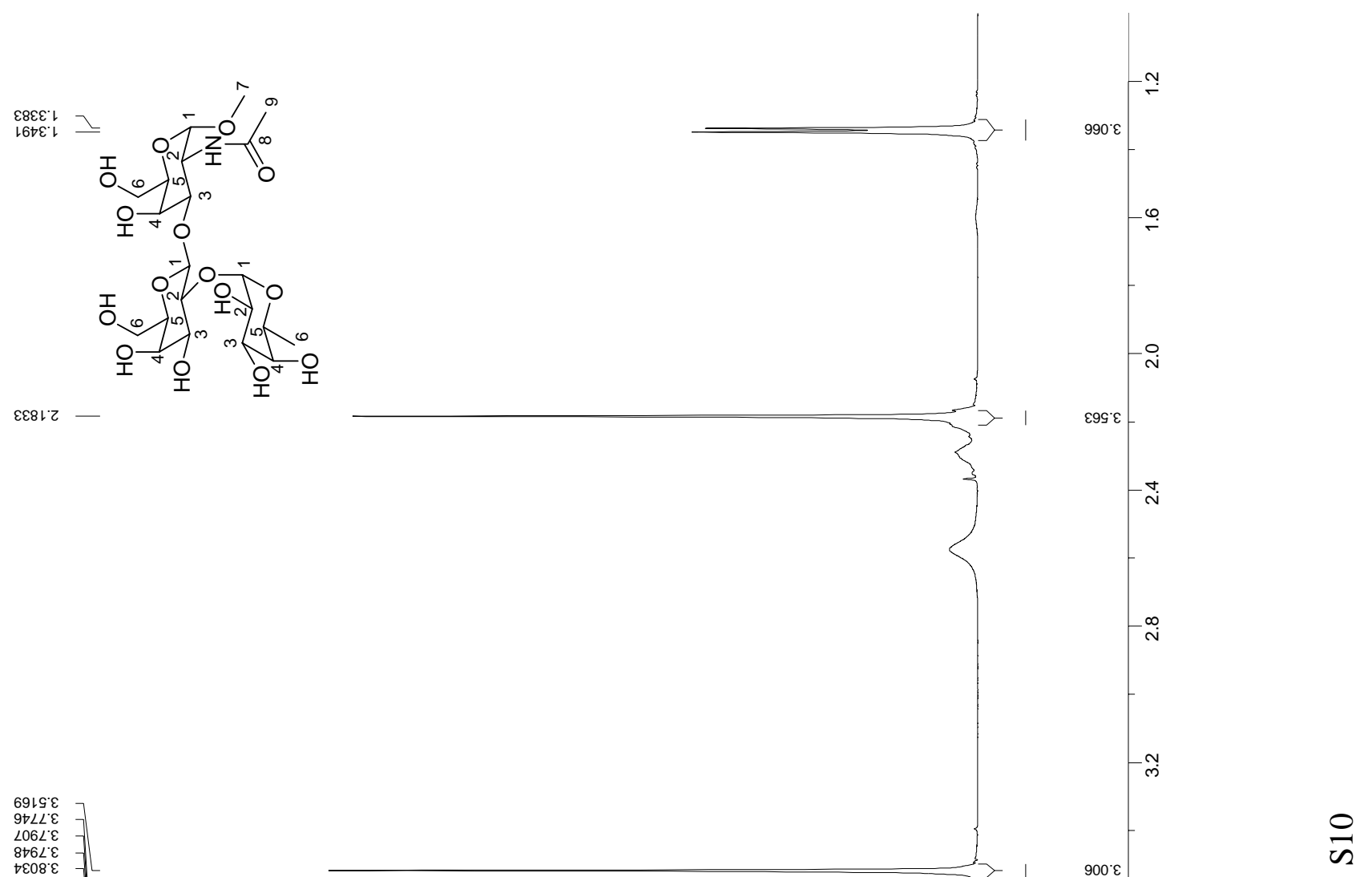

0

1

$\#$

న

포

8

을

클

굴

赵

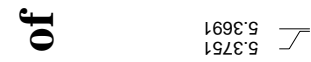

$691 c^{\prime} \varepsilon$

$9 \nabla L L \cdot \varepsilon$
$\angle 06 L^{\prime} \varepsilon$

$8 \nabla 6 L \mathcal{E}$
$\nabla \varepsilon 08^{\circ} \varepsilon$

$\neg \angle 18^{\circ} \varepsilon$

टटट८ $\varepsilon$

†เ88 $\varepsilon$

$\varepsilon+68^{\circ} \varepsilon$

$6+06^{\circ} \varepsilon$

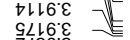

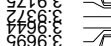

†086 $\varepsilon$

$9986^{\circ} \varepsilon$

0290 $\circ$

$69900^{\circ} \circ$

0860' $t$

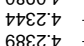

0ह९ट"

$\forall \angle S Z{ }^{\prime} \triangleright$

$0 \angle Z \varepsilon " \nabla$

$S+\varepsilon " t$

caget

999't -

$\angle 98 \angle ' t$

$2 \varepsilon 266^{\circ} t$

$826^{\circ} t$

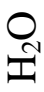

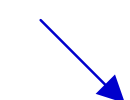

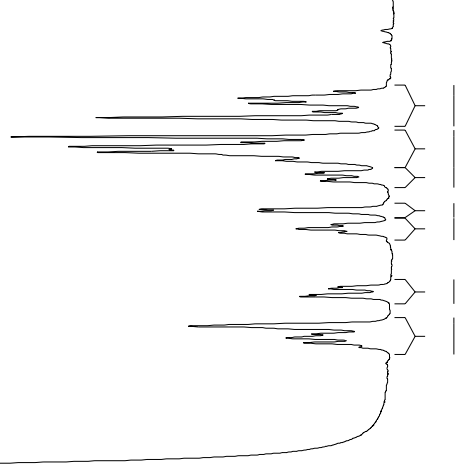

-

こเที

Isto

เZZ'

S60.t

$\angle 60.5$

9028

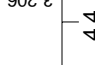

$\downarrow \varepsilon \varepsilon^{\prime} \downarrow-\infty$

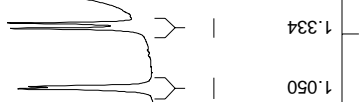

ก

$000^{\circ}$

$\sum_{\substack{a \\ a}}$ 
$9\llcorner\varepsilon \varepsilon \Im \vdash$

$90 \varepsilon 6$.

L06E'6t

เจ80 99

เยเย.6S

1206.09
$08+2.19$

टहLL.99 -

$\angle 190.89-$

$99+0.69-$

$98 \nabla 9.69$

$2 L 8 E .0 L$
$0 E+8.1 L$

$\angle 8 \varepsilon G^{\circ} \varepsilon \angle$

OSGL'EL

टथLट ${ }^{\circ} 9 L$

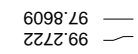

1996.101 -

ข

f

స

刃

อิ

옹

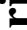

$\frac{2}{2}$

e

블

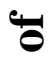

ㄴ

$\sum_{Z}$

?

$6609^{\circ} \varepsilon \angle t$

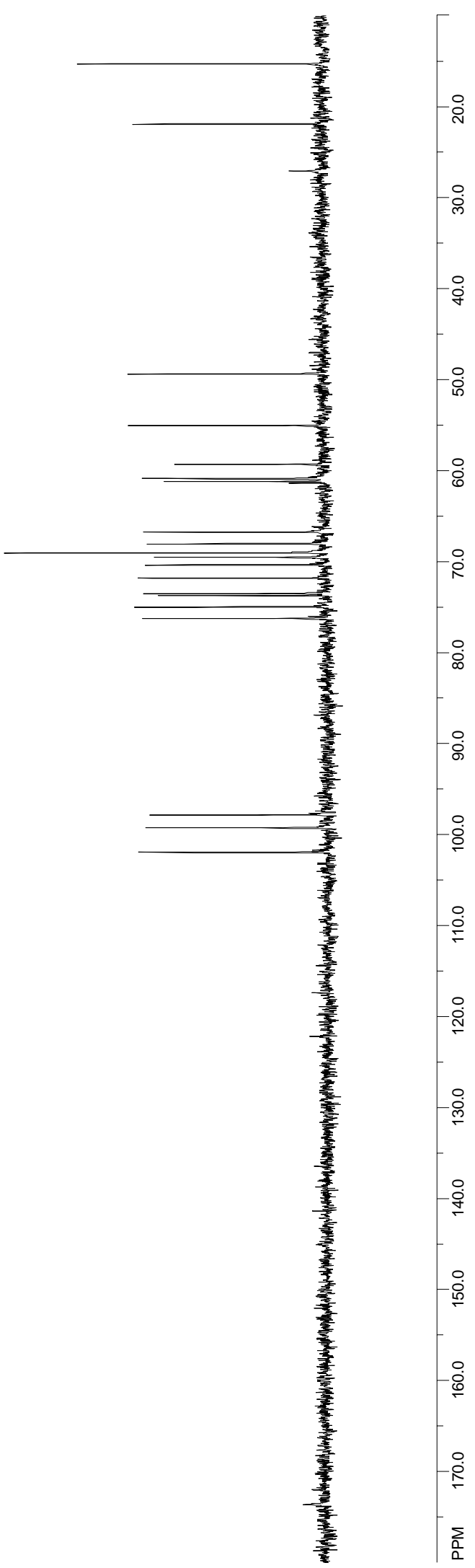

$\overline{\text { s }}$

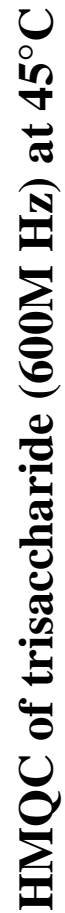

i 


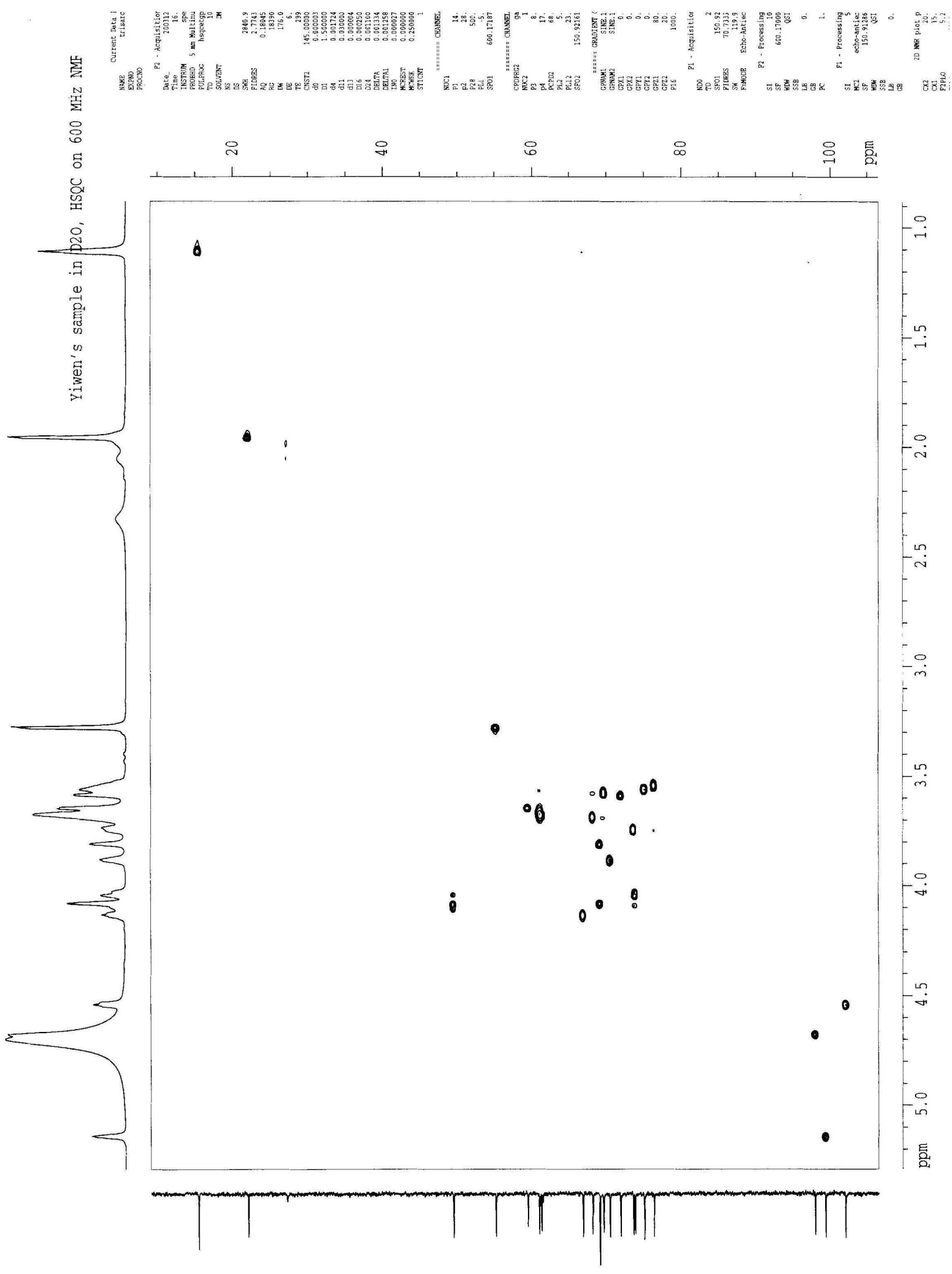



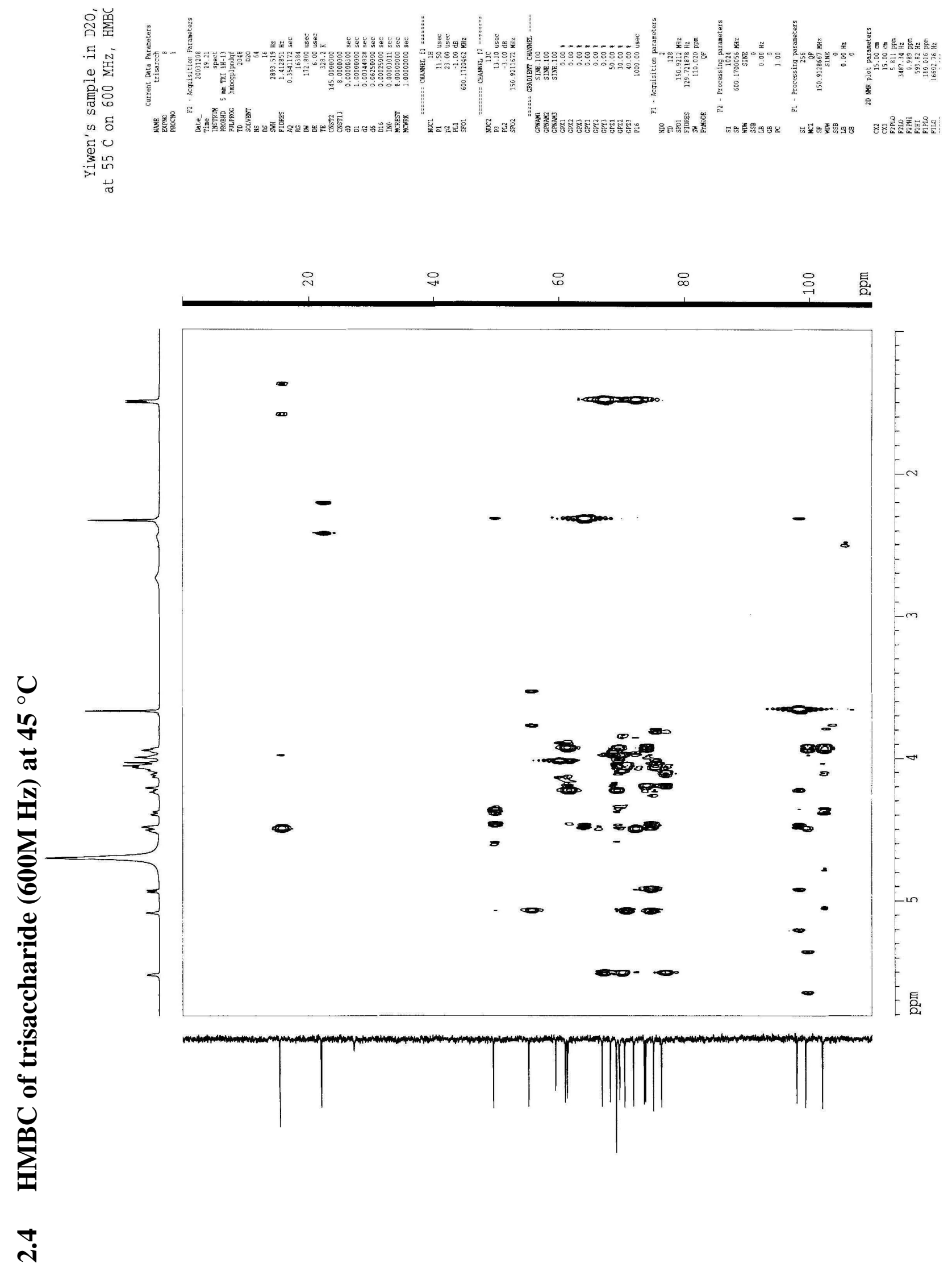

$\frac{m}{n}$ 


\section{Tetrasaccharide $\alpha$-L-Fuc $p$-[ $\alpha$-D-Gal $p(1,3)](1 \rightarrow 2)-\beta$-D-Gal $p-(1 \rightarrow 3)$ - $\alpha$-Gal $p$ NAc-OMe}

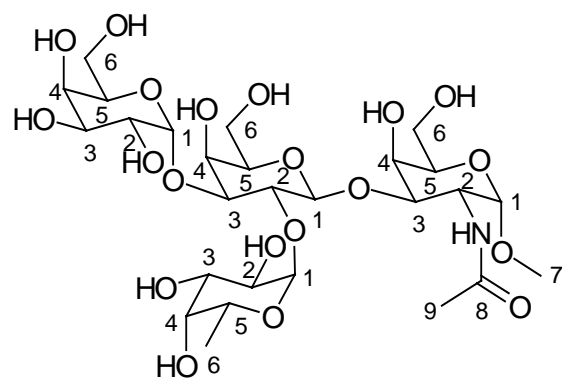

Exact Mass $(\mathrm{M}+\mathrm{Na})+: 728.2583$

Found: 728.2584

\begin{tabular}{|c|c|c|c|c|c|c|c|c|}
\hline & \multicolumn{2}{|c|}{ GalNAc } & \multicolumn{2}{|l|}{$\beta$-Gal } & \multicolumn{2}{|l|}{$\alpha$-Gal } & \multicolumn{2}{|l|}{ Fuc } \\
\hline \# & ${ }^{1} \mathrm{H}(\delta)$ & ${ }^{13} \mathrm{C}(\delta)$ & ${ }^{1} \mathrm{H}(\delta)$ & ${ }^{13} \mathrm{C}(\delta)$ & ${ }^{1} \mathrm{H}(\delta)$ & $\begin{array}{l}{ }^{13} \mathrm{C} \\
(\delta)\end{array}$ & ${ }^{1} \mathrm{H}(\delta)$ & $\begin{array}{l}{ }^{13} \mathrm{C} \\
(\delta)\end{array}$ \\
\hline 1 & $\begin{array}{l}4.73, \mathrm{~d}, J=3.3 \\
\mathrm{~Hz}, 1 \mathrm{H}\end{array}$ & 98.20 & $\begin{array}{l}4.65, \mathrm{~d}, J=7.5 \\
\mathrm{~Hz}, 1 \mathrm{H}\end{array}$ & 102.56 & $\begin{array}{l}5.20, \mathrm{~d}, J=3.3 \\
\mathrm{~Hz}, 1 \mathrm{H}\end{array}$ & 93.41 & $\begin{array}{l}5.21, \mathrm{~d}, J=4.5 \\
\mathrm{~Hz}, 1 \mathrm{H}\end{array}$ & 99.25 \\
\hline 2 & $4.10, \mathrm{~m}, 1 \mathrm{H}$ & 49.74 & $3.96, \mathrm{~m}, 1 \mathrm{H}$ & 73.32 & $3.84, \mathrm{~m}, 1 \mathrm{H}$ & 68.51 & $3.72, \mathrm{~m}, 1 \mathrm{H}$ & 68.10 \\
\hline 3 & $3.70, \mathrm{~m}, 1 \mathrm{H}$ & 63.87 & $3.92, \mathrm{~m}, 1 \mathrm{H}$ & 76.50 & $3.92, \mathrm{~m}, 1 \mathrm{H}$ & 69.88 & $\begin{array}{l}3.55, \mathrm{dd}, J=7.8, \\
3.2 \mathrm{~Hz}, 1 \mathrm{H}\end{array}$ & 70.38 \\
\hline 4 & $3.61, \mathrm{~m}, 1 \mathrm{H}$ & 72.25 & $4.15, \mathrm{~m}, 1 \mathrm{H}$ & 69.57 & $4.08, \mathrm{~m}, 1 \mathrm{H}$ & 69.73 & $3.61, \mathrm{~m}, 1 \mathrm{H}$ & 75.00 \\
\hline 5 & $3.83, \mathrm{~m}, 1 \mathrm{H}$ & 70.73 & $4.10, \mathrm{~m}, 1 \mathrm{H}$ & 74.66 & $4.23, \mathrm{~m}, 1 \mathrm{H}$ & 71.50 & $4.22, \mathrm{~m}, 1 \mathrm{H}$ & 67.27 \\
\hline 6 & $3.75, \mathrm{~m}, 2 \mathrm{H}$ & 61.35 & $3.74, \mathrm{~m}, 2 \mathrm{H}$ & 61.65 & $3.73, \mathrm{~m}, 2 \mathrm{H}$ & 61.76 & $\begin{array}{l}1.47, \mathrm{~d}, J=6.6 \\
\mathrm{~Hz}, 3 \mathrm{H}\end{array}$ & 15.73 \\
\hline 7 & $\begin{array}{l}3.32, \mathrm{~s}, 3 \mathrm{H} \\
\left(\mathrm{OCH}_{3}\right)\end{array}$ & 55.52 & & & & & & \\
\hline 8 & $\mathrm{C}=\mathrm{O}$ & 174.09 & & & & & & \\
\hline 9 & \begin{tabular}{|l|}
$2.01, \mathrm{~s}, 3 \mathrm{H}$ \\
$\left(\mathrm{COCH}_{3}\right)$
\end{tabular} & 22.42 & & & & & & \\
\hline
\end{tabular}




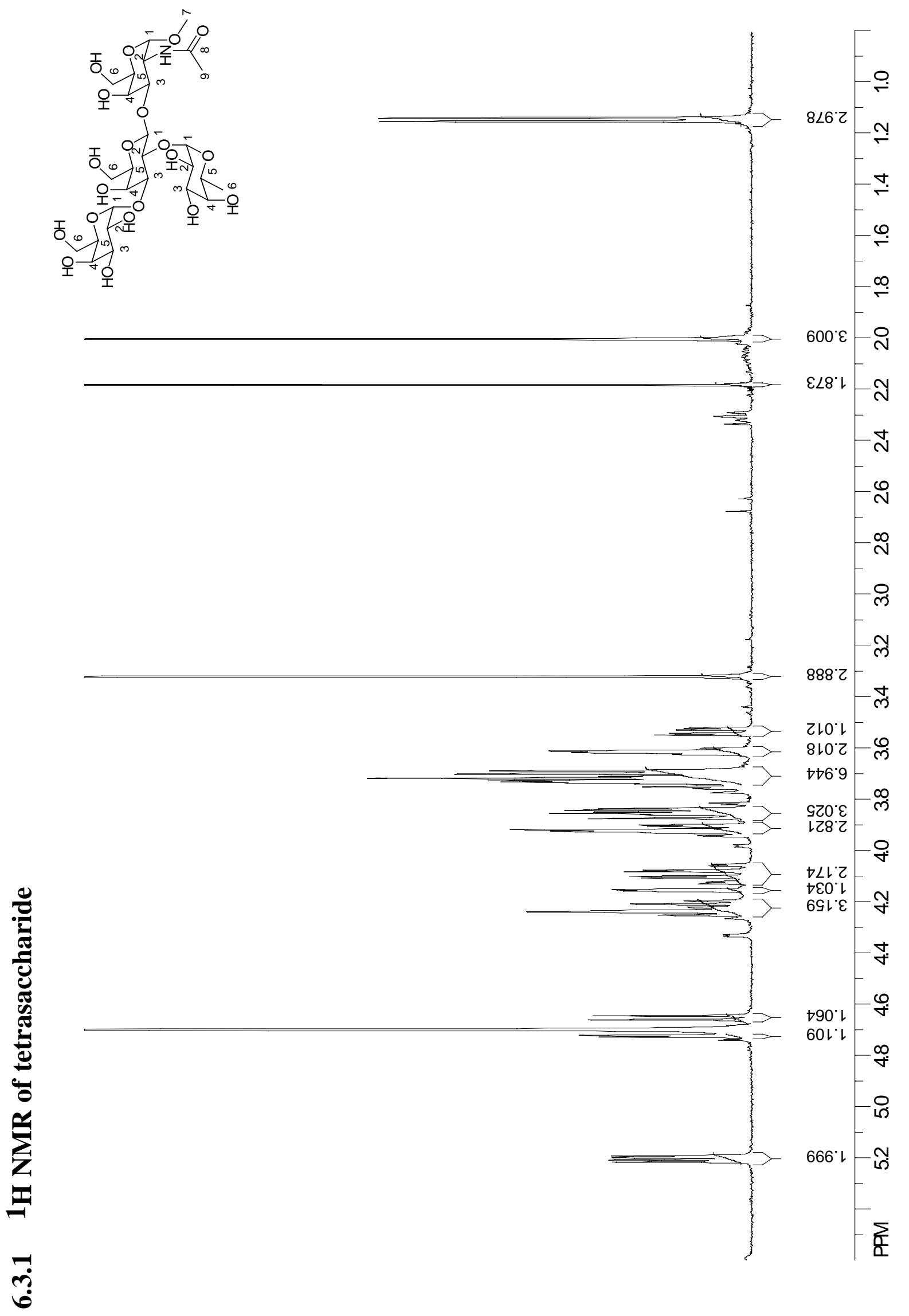




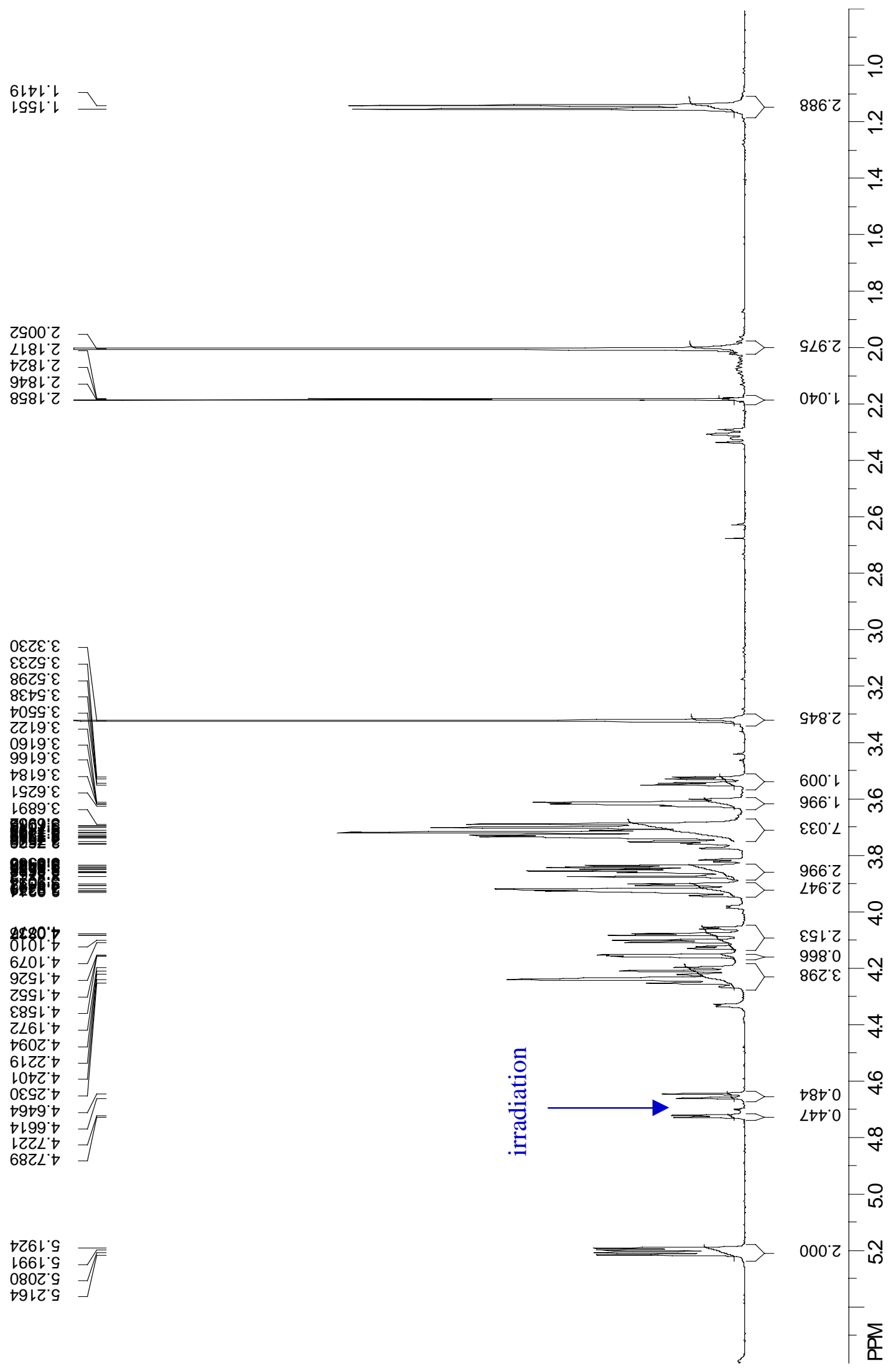

กั. 

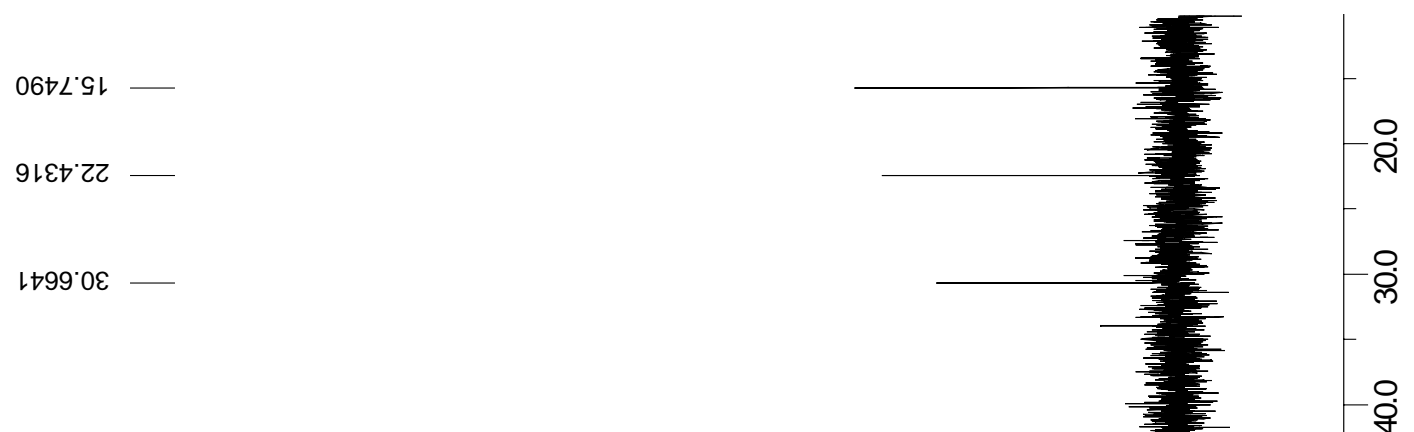

$9 t S L \cdot 6 t$

08ES. 99

$0+L \varepsilon \cdot 19$

LCLL' I9

$\angle 288^{\circ} \mathrm{E} 9$

$6+82: \angle 9$

9ट1เ.89

†0\&9. 89

0989.69

$00 t \angle \cdot 69$

$669 L$ L 69

.

ऽE6E.0L

9919.1

1892' $2 L$

8เEย' $\varepsilon L$

$86 \angle 9^{\circ} \rightarrow L$

8110.92

เZZナ'E6 -

$\varepsilon 01 Z^{\prime} 86$

0 เ9Z'66

SOLG'ZO

อิ

g

䓪

2

こ๐トレLL -

ตุ? 


\subsubsection{2-D NMR spectra of tetrasaccharide}
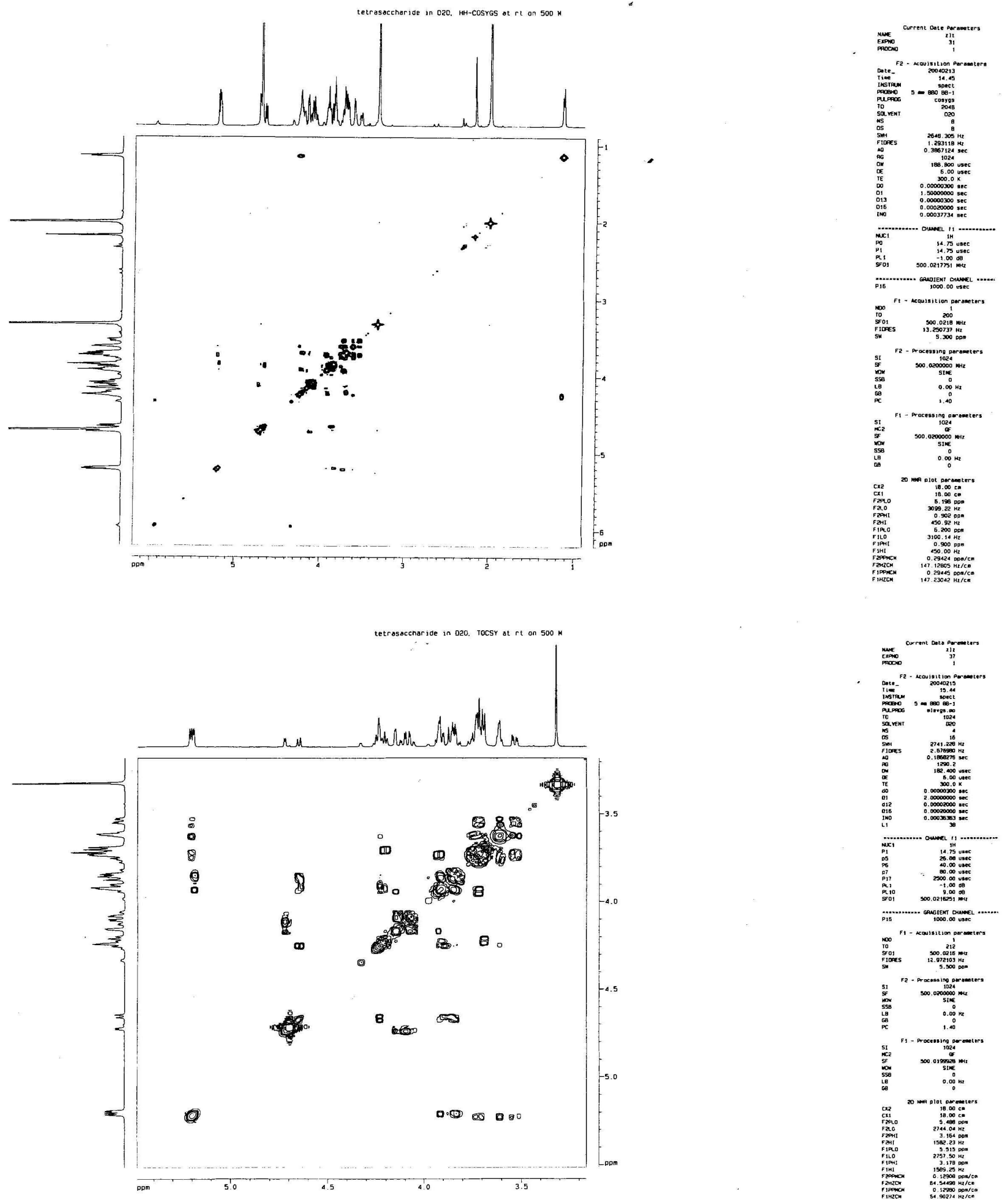